\title{
ILCEA
}

Revue de l'Institut des langues et cultures

d'Europe, Amérique, Afrique, Asie et Australie

28 | 2017

Passages, ancrage dans la littérature de voyage

\section{De Europa et Asia d'Enea Silvio Piccolomini : migrations, invasions, ancrages, un état des lieux de l'Europe au XV siècle}

De Europa and Asia by Enea Silvio Piccolomini: Migrations, Invasions, Points of Anchorage, an Inventory of Europe During the 15th Century.

\section{Serge Stolf}

\section{(2) OpenEdition}

\section{Journals}

Édition électronique

URL : http://journals.openedition.org/ilcea/4071

DOI : 10.4000/ilcea.4071

ISSN : 2101-0609

Éditeur

UGA Éditions/Université Grenoble Alpes

Édition imprimée

ISBN : 978-2-84310-374-2

ISSN : 1639-6073

Référence électronique

Serge Stolf, «De Europa et Asia d'Enea Silvio Piccolomini : migrations, invasions, ancrages, un état des lieux de l'Europe au XVe siècle », ILCEA [En ligne], 28 | 2017, mis en ligne le 06 mars 2017, consulté le 19 avril 2019. URL : http://journals.openedition.org/ilcea/4071 ; DOI : 10.4000/ilcea.4071

Ce document a été généré automatiquement le 19 avril 2019

(C) ILCEA 


\title{
De Europa et Asia d'Enea Silvio Piccolomini : migrations, invasions, ancrages, un état des lieux de l'Europe au XV siècle
}

\author{
De Europa and Asia by Enea Silvio Piccolomini: Migrations, Invasions, Points of \\ Anchorage, an Inventory of Europe During the 15th Century.
}

\section{Serge Stolf}

1 Comme l'indique son titre, cette brève contribution se donne pour objet un examen des «lieux» de l'Europe, tels qu'un humaniste $\mathrm{du} \mathrm{Xv}^{\mathrm{e}}$ siècle - Enea Silvio Piccolomini (1405-1464) - les présente dans deux ouvrages latins, De Europa (L'Europe) et Asia (L'Asie), écrits respectivement en 1458 et en 1461. Dans l'un et dans l'autre, le propos n'est pas seulement géographique et historique, mais aussi largement culturel. Aussi la question des « lieux » est-elle envisagée du point de vue, essentiellement humain, des mouvements de populations, des passages et des ancrages, dans le but d'offrir de l'Europe non une image figée dans un moment particulier de son histoire, mais saisie dans sa réalité provisoire et instable. Tout en faisant de cette réalité une analyse en quelque sorte «à chaud" - dans l'urgence d'une situation d'affrontement entre l'Europe et les Ottomans -, l'auteur soulève des problèmes de frontières et d'identités, qui pour être résurgents, aujourd'hui, dans un espace dont les contours apparaissent plus que mouvants, ne sont évidemment identiques qu'en apparence, comme le soulignait Federico Chabod à propos des problèmes historiques (Europes, $2000: 211$ ).

2 Quand la constitution européenne insère dans son préambule la mention des « héritages culturels, religieux et humanistes de l'Europe » (Traité de Lisbonne, 2009) et quand les autorités turques donnent une solennité toute particulière au $562^{\mathrm{e}}$ anniversaire de la conquête de Constantinople (30 mai 2015) - pour ne choisir que deux grands protagonistes des analyses de Piccolomini - on remarque une permanence des points d'ancrage (même s'ils apparaissent de manière générique dans la constitution 
européenne, sans référence à des identités précises) dans une profondeur historique évidente, qui sont non seulement politiquement, mais culturellement identifiables, et dont la littérature elle-même s'est emparée, entre réalités et mythes (Ossola, 2001).

Quelle perception un lettré européen du $\mathrm{xv}^{\mathrm{e}}$ siècle comme Enea Silvio Piccolomini a-t-il eu de ces migrations-invasions qui marquent son temps? Lui-même, siennois d'origine, «italien» passé en terre allemande, où il servit pendant une quinzaine d'années les intérêts diplomatiques et politiques de l'empereur Frédéric III de Habsbourg dans le même temps où il entamait une carrière ecclésiastique - elle le conduira au pontificat en 1458 - fut un infatigable voyageur, ses pérégrinations souvent imposées par ses fonctions l'amenant à une connaissance sur le terrain, quand elle n'était pas livresque, des peuples et des populations. S'il s'est constamment déplacé - en Écosse, en Angleterre, en terres d'Empire, en Hongrie, en Bohême et en Italie -, son ancrage demeure l'humanisme, la culture classique profane et chrétienne, convaincue de la vocation universelle, et universaliste, de ses valeurs. Les deux ouvrages cités, qui connurent au $\mathrm{xVI}^{\mathrm{e}}$ siècle de multiples éditions, demeurent peu connus dans le domaine français, ni l'un ni l'autre n'y étant encore traduits, contrairement aux éditions du De Europa en allemand (Piccolomini, 2005) ou de Asia en italien (Piccolomini, 2004) et en espagnol (Piccolomini, 2010).

Piccolomini, qui est cardinal de Sainte-Sabine depuis décembre 1456, écrit en 1458 le De Europa, ouvrage d'actualité décrivant l'Europe de son temps sous ses aspects essentiellement humains et géopolitiques : une géographie des lieux et des cultures. Il ne s'agit pas à proprement parler d'une chorographie; les milieux et les paysages ne l'intéressent ici qu'assez peu. Le propos général, qui passe en revue toutes les nations et les régions d'Europe, s'efforce de définir les traits qui caractérisent et différencient les Européens de ceux qui ne le sont pas. Il emploie pour la première fois l'adjectif latin, Europeus : «Europeos homines » (Piccolomini, $2001: 27$ ), au lieu d'un substantif plus ancien, Europenses (Grattarola, $1986^{1}$ : 174-186). Le vocabulaire permet ici de percevoir une définition reposant sur l'appartenance à une communauté humaine, identifiable par des traits communs, peuplant une entité géographique aux limites circonscrites. Dans un contexte marqué par la pression exercée depuis la fin du XIV e siècle par les Ottomans sur les frontières de l'Europe, qu'ils ont déjà franchies, et par la prise de Constantinople en mai 1453 qui met fin à l'Empire romain d'Orient, les non-Européens sont essentiellement les Turcs et les peuples d'Asie dont ils sont originaires. C'est en 1461, alors qu'il est pape (Pie II) depuis trois ans que Piccolomini rédige l'Asia, description historique et géographique de ce continent, fondée sur des sources indirectes et essentiellement livresques (les géographes de l'Antiquité), et de fait moins renseignée que le De Europa.

Les liens thématiques entre les deux ouvrages, comme je le montrerai, sont nombreux: l'Asie est un réservoir de populations nomades qui, dans un mouvement de l'Est - ou de l'Orient - vers l'Occident, a submergé et submerge l'Europe. Pie II les décrit selon des critères de type anthropologique, moral et culturel, en les situant sur une échelle de plus ou moins grande humanité : des termes comme «cette race d'hommes cruels » (id genus immanium hominum), "peuple aux mœurs répugnantes" (gens foeda) reviennent fréquemment. Ces peuples, dont Pie II rapporte les mœurs sans aucune référence à des récits de voyageurs contemporains, et souvent sur la foi d'historiens antiques, sont situés au-delà d'une frontière où commence l'inexploré. Ce sont donc souvent les « mœurs » ou «modes de vie » (mores) et l'erratisme de ces populations qui les différencient de celles d'une Europe identifiée comme le «lieu», privilégié par une longue tradition - une conquête aussi - de culture latine et chrétienne, d'une humanitas - la réalisation que doit 
poursuivre l'homme pour parfaire sa nature, en terme de morale, de sociabilité et de capacités créatives ${ }^{2}-$, mot qui pourrait recouvrir ce que nous appellerions un degré de civilisation :

Or, si nous croyons qu'il y a réellement eu un grand changement dans les mœurs par rapport à celles rapportées par les Anciens - selon lesquels les Scythes étaient presque tous bergers et à peu près tous intraitables - et que l'âpre contrée nordique n'admet pas ces mœurs qui ont fait resplendir autrefois la Grèce et aujourd'hui font briller l'Italie, ce Cathay qu'ils louent si fort est certainement situé moins au nord que ne le montrent les cartes ${ }^{3}$. (Piccolomni, $2004: 41$, § 15)

6 Les frontières ne sont donc pas celles qui séparent les peuples et que dessine la géographie (les déserts sont traversés, les fleuves franchis), mais elles se définissent par le degré d'assimilation et de sédentarisation, d'ancrages dans cette humanitas. Il est donc ici question de lieux, de territoires, d'itinéraires, qui ne sont pas neutres : les migrations peuvent être accompagnées de violences, se transformer en occupations de territoires et susciter des résistances dont l'enjeu est la vitalité même de la civilisation. Ainsi, les «barbares » peuvent être en Europe, mais en être exclus culturellement. Au point qu'à l'intérieur même de l'espace européen, un Italien comme Piccolomini juge les Allemands «barbares » parce qu'ils ne sont pas encore passés à la culture humaniste. Je me propose d'examiner ces questions en deux moments: le premier concernera le rapport EuropeAsie, sous l'aspect des passages, des migrations, des invasions, en mettant en évidence les phénomènes d'extension et de contraction; le second sera centré sur les ancrages culturels de l'Europe et sur ses résistances à l'altérité.

\section{Le rapport Europe-Asie}

Commençons par une simple notation géographique : Europe et Asie sont séparées par le Bosphore, «seul espace séparant l'Europe de l'Asie ${ }^{4}$ » (Piccolomini, $2001:$ 71), un espace qui divise deux continents, mais qui, après avoir été le lieu de passage des Grecs (plus tard romanisés) vers l'Asie Mineure, devient au xve siècle le lieu de passage en sens inverse des envahisseurs turcs pour la conquête de Constantinople et celle des Balkans, déjà amorcée. Or, l'Europe, pour Piccolomini, se définit comme l'expansion de la romanité chrétienne, celle-ci fût-elle en territoire géographiquement asiatique et de langue grecque. Aux lettrés ou historiens qui attribuent aux Turcs une ascendance troyenne parce qu'ils occupent l'Asie Mineure, il répond qu'ils ne peuvent être considérés comme les héritiers d'une race et d'une civilisation originaires d'Europe, ni se vanter d'une telle ascendance : «Les Troyens étaient originaires de Crète et d'Italie; la race des Turcs est scythe et barbare ${ }^{5}$. (Piccolomini, 2001 : 62-63)

8 La chute de Constantinople et, partant, de l'empire byzantin va être perçue par Piccolomini comme une occupation et un rétrécissement en termes de culture de l'espace européen. C'est ici que l'on peut parler de phénomène de contraction. Il insiste particulièrement sur le sort réservé aux églises et aux bibliothèques, dans une lettre du 12 juillet 1453 au pape Nicolas V :

Je souffre à la pensée des nombreuses basiliques consacrées aux saints, constructions d'un admirable travail, soumises à la destruction ou à la souillure de Mahomet. Que dirais-je des livres innombrables qui se trouvaient en ce lieu, encore inconnus aux Latins? Las, combien de noms de grands hommes périront à présent? C'est la seconde mort d'Homère, le second trépas de Platon. Où rechercherons-nous les œuvres de génie des philosophes et des poètes? La source des Muses est tarie, 
puisse-t-il nous rester assez de talent pour pouvoir pleurer ce grand malheur avec de dignes accents ${ }^{6}$. (Wolkan, $1918: 200$ )

Constantinople avait été un lieu d'ancrage de la culture grecque, mais aussi celui du passage de cette culture vers l'Italie. Il est à peine besoin de souligner le rôle joué par les voyages accomplis, dès le tout début $d u \mathrm{xv}^{\mathrm{e}}$ siècle, par des lettrés se rendant d'Italie à Constantinople pour y acquérir des manuscrits grecs ignorés par les lecteurs occidentaux: ouvrages techniques et scientifiques, d'histoire, de géographie et de philosophie. Le sac de la capitale byzantine et la destruction de cent vingt mille ouvrages de la bibliothèque impériale ${ }^{7}$ accentuent un mouvement inverse, mais d'exil à présent, des lettrés byzantins vers l'Italie où beaucoup s'étaient déjà rendus, favorisant ainsi la diffusion de la langue et de la littérature grecques dans les milieux humanistes de la péninsule. Piccolomini est conscient qu'il n'y aura pas de retour vers la source, que le transfert d'une partie de cette culture s'opérera vers Rome, certes, c'est-à-dire vers l'Europe - dont le patrimoine pourrait bien être encore menacé de disparition si rien n'est fait pour le défendre et le protéger - mais il n'en reste pas moins qu'une perte, dans ce patrimoine universel, demeure irréparable. On mesure alors combien les ancrages sont fragiles, faits de retours vers la "barbarie », de sauvegardes improbables dans un espace désormais abandonné au vandalisme et aux amputations.

10 Ainsi, la région que Piccolomini appelle Romania (Roumanie) - en fait la Roumélie, toute la zone maritime jusqu'à l'Hellespont (détroit des Dardanelles) correspondant à la Thrace et à la Macédoine - est une parcelle d'Europe revenue à son état originel d'avant la civilisation : « [Elle est] de nation grecque, bien qu'autrefois elle ait été barbare, et que, de nos jours, après la destruction de l'empire grec, sous la domination des Turcs, elle soit retournée à la barbarie ${ }^{8}$.» (Piccolomini, 2001 : 59). Le sort de la Roumélie, terre romaine peuplée de Grecs, montre qu'une autre séparation intervient désormais, qui transgresse ces frontières physiques puisque l'Europe occupée par les Turcs n'est plus l'Europe, mais le territoire des barbares. Le berceau de la civilisation, Athènes, n'est plus : Enea note la prise de l'Acropole, en juin 1458 (Piccolomini, 2001 : 88).

11 Ce point de vue conditionne l'interprétation qu'il donne de ces migrations de l'Asie vers l'Europe : celles-ci relèvent de ce nous avons appelé plus haut " phénomène d'extension ». La partie septentrionale de l'Asie [les steppes de l'Asie centrale, au nord de la mer Noire] est peuplée de nations caractérisées par leur nomadisme, forme de vie associée à l'absence de villes et de vie politiquement organisée. Piccolomini est convaincu que les zones septentrionales - effet de la rudesse du climat? - sont autant prédisposées à la barbarie - «la nation des Scythes est barbare9 ( (Piccolomini, 2004: 66, § 28) - que les contrées méridionales le sont à la civilisation. C'est l'antique pays des Scythes, décrits par Hérodote, et associé à la sauvagerie des mœurs. Pour Piccolomini, la Scythie est le pays d'où sont venues toutes les hordes d'envahisseurs qui déferlèrent sur l'Occident: Huns d'Attila, Goths, Lombards, Hongrois, Turcs enfin, ultime avatar des Scythes ${ }^{10}$ (Piccolomini, 2001 : 68-70). Les verbes qu'il emploie à propos du déferlement des Huns sont significatifs du lien établi - migration-occupation-invasion-destruction :

De la Scythie, en Asie, émigrèrent (migraverunt) vers l'Europe les Huns, peuples féroces $[. .$.$] qui [...] occupèrent (occupavere) toute la contrée barbare comprise entre$ le Tanaïs [le Don] et le Danube. Né parmi ces gens-là, le grand Attila, après avoir soumis la Pannonie, ayant pénétré (ingressus) en Italie, ruina Aquilée et, passant ( transiens) en Allemagne, il y commit de nombreuses dévastations, puis, ayant franchi (transmisso) le Rhin... » (Piccolomini, $2004: 68, \S 29)^{11}$ 
12 L'Asia reflète des préoccupations et des anxiétés tout à fait contemporaines. La description de l'Asie et son histoire se configurent comme celles d'un continent demeurant totalement étranger à l'Europe. Les grands mouvements que note Piccolomini sont précisément ceux des migrations vers l'Occident, comme si le continent asiatique était le réservoir d'une barbarie prête à déferler sur la civilisation ${ }^{12}$. On constate la modernité de la liaison qu'il établit entre géographie, modes de vies et histoire, et l'attention qu'il porte aux "changements " (le mot mutationes revient fréquemment) provoqués par ces incessantes poussées de populations de l'Asie vers l'Europe: dans le contexte où il les observe, l'avancée turque est le prélude d'une colonisation - effective dans les Balkans -, d'un enracinement de peuples étrangers et hostiles à la culture européenne.

\section{L'Europe comme ancrage et ses résistances}

13 Piccolomini définit deux points d'ancrage principaux pour l'Européen qu'il est : la culture héritée du monde gréco-romain et celle héritée du christianisme. Ce sont là les vrais ports d'attache de ce qu'il définit avant tout comme une communauté : il en parle comme de «notre patrie, [de] notre propre maison ${ }^{13}$ " (Opera omnia, 1551: 678). Au-delà de la conception spatiale, des délimitations linguistiques, des entités politiques ou ethniques, c'est des « européens » qu'il entend parler. Les frontières physiques sont très incertaines vers l'Est et vers le Nord, là où les terrae incognitae (« terres inconnues ») ont reculé, sans disparaître, mais sont à présent remodelées sous l'effet de la christianisation. À la périphérie de cet espace, il constate que les lieux qui ont été ceux des passages des envahisseurs du $\mathrm{v}^{\mathrm{e}}$ siècle, sont devenus des lieux de sédentarisation et d'acculturation. Les Hongrois, peuple d'origine asiatique, des Scythes peut-être, ont franchi le Don et se sont installés sur les deux rives du Danube (Ister) en en chassant les populations ou en les soumettant. Cependant, Piccolomini distingue deux Hongries :

Il existe encore, non loin de la source du Don [Tanaïs], une autre Hongrie, souche de la nôtre [...] qui lui est presque semblable par la langue et les coutumes, bien que la nôtre soit plus civilisée, adorant le Christ ; celle-là vivant à la manière des barbares est l'esclave des idoles ${ }^{14}$. (Piccolomini, $2001: 29$ )

14 De même indique-t-il que :

Le nord de la Valachie est occupé par les Roxani, que nous appelons de nos jours les Ruthènes [au sud de l'Ukraine actuelle], et vers le fleuve Dniestr vivent des nomades, de race scythe, que nous appelons aujourd'hui Tartares. [...] Le peuple y parle encore la langue de Rome, bien qu'elle soit en grande partie changée et à peine intelligible pour un italien ${ }^{15}$. (Piccolomini, $2001: 57$ )

Le nomadisme appartient à l'Asie, origine des grandes migrations vers l'Occident, dessinant les frontières de l'Europe et de ses peuples sédentarisés. En deçà de ces limites sud-orientales, nous sommes en pays de langue latine ou romane (le roumain), c'est-àdire dans l'espace de l'histoire, là où l'empire romain marqua son expansion jusqu'au pays des Daces.

16 Si ces limites naturelles du continent le dessinent comme un contenant, l'Europe se définit et s'identifie par un autre espace, celui des peuples qui l'occupent, l'espace d'une histoire construite en commun. Ce qui le caractérise, ce sont les migrations, de l'Orient vers l'Occident, des populations nomades qui, en se sédentarisant, ont configuré le paysage humain de l'Europe. L'histoire est définie par le mouvement, le « changement ». 
Le terme de mutatio revient à plusieurs reprises ${ }^{16}$, comme nous l'avons souligné plus haut, sous la plume de Piccolomini. Le caractère mouvant et fluctuant des constructions humaines justifie le projet même de Piccolomini, explique le regard porté par l'historien qu'il est sur les plus récentes de ces mutations qui ont affecté l'Europe :

Étonnante mutation des choses et gloire éphémère de la domination de l'homme: voici la Macédoine, illustre sous deux rois, et qui, ayant assujetti la Grèce et la Thrace, étendit son empire sur l'Asie [...]. La voici, la même, soumise aujourd'hui à cette immonde race des Turcs, obligée de payer tribut et de supporter un misérable joug $^{17}$. (Piccolomini, $2001: 86$ )

Inversement, l'Europe est traversée par les phénomènes de mobilité très dynamiques que sont l'évangélisation des terres nordiques d'une part, et le mouvement humaniste d'autre part. Lorsqu'il dit qu'un Italien ne comprend presque rien de la langue des Ruthènes, malgré ses racines latines, Piccolomini entend désigner le fonds commun d'une culture dont l'Italie et Rome sont les héritiers et dont la responsabilité est celle d'un apostolat, que nous pourrions définir " laïc ", de l'humanisme. L'évangélisation, en convertissant les peuples baltes au christianisme, a libéré, en partie, ces peuples de leurs superstitions : leur christianisation permet donc de les assimiler aux Européens. C'est ainsi qu'il définit la Livonie (territoires actuels de la Lettonie et de l'Estonie) comme «la dernière des provinces chrétiennes $»^{18}$ (Piccolomini, $\left.2001: 118\right)$ : «La religion chrétienne ouvrit cette partie de la terre à notre race, montrant à ses peuples particulièrement féroces, une fois purgée leur barbarie, un genre de vie plus doux ${ }^{19}{ }$ (Piccolomini, 2001 : 118-119). Il est intéressant de noter que cette évangélisation «a ouvert » (aperuit) de nouveaux espaces en intégrant des peuples barbares à la Chrétienté, à la « race », nation ou communauté européenne.

$18 \mathrm{Au}$ sud, Grenade constitue l'extrême limite de cet espace. Ce royaume «hostile à l'Évangile du Christ $»^{20}$ (Piccolomini, 2001 : 187) est cependant terre d'Europe, car il est sous-entendu qu'elle est promise à la reconquête. Le lexique utilisé, Hispani pour désigner les populations chrétiennes de l'Espagne, Saraceni (Piccolomini, 1991: 87) pour celles du royaume de Grenade, montre que, dans le contexte de l'époque, Piccolomini considère ces derniers, adeptes de Mahomet, comme une présence hostile, au même titre que celle des Turcs, au sein d'une Europe chrétienne, et qu'il convient d'extirper. Il souligne le rôle éminent des souverains de Castille dans cette Reconquista dont une des dernières étapes, de son vivant, est la prise de Gibraltar en 1462 par les armées d'Henri IV de Castille. Cette « reconquête » [il emploie le verbe latin recuperare] a une valeur symbolique :

C'est de nos jours seulement que Gibraltar est de nouveau entre des mains chrétiennes, et que, remplaçant les temples de Mahomet, des églises ont été consacrées à la Piété divine et que de nombreux habitants chrétiens ont afflué là [en masse] ${ }^{21}$. (Piccolomini, 1984 : livre X, 616-617)

19 Et nous avons là ce que pourrait représenter un port d'attache. Piccolomini dira à propos de l'islamisation de la Bosnie, en substance, que si l'on n'est pas assez ancré dans sa foi et dans sa culture, celles-ci disparaissent. De même, il commence la description de l'Europe par la Hongrie parce que celle-ci représente alors le point d'ancrage, ou le rempart, le plus fort dans la résistance aux Ottomans, un avant-poste de cette lutte vitale pour l'Europe assiégée : « Les Hongrois [qui] jusqu'ici ont été le bouclier de notre foi, le mur de notre religion ${ }^{22}$.» (Opera omnia, $1551: 682$ )

$\mathrm{Au}$ terme de ce bref excursus dans la «terre étrangère [du passé] » (Lowe, 2013), on constate l'importance revêtue pour Piccolomini par les notions de passages et d'ancrages. Le projet d'une géographie entraîne avec lui une réflexion sur le caractère mouvant des 
frontières qui, au-delà de leur définition strictement politique, peuvent devenir des lignes d'expansion ou des murs, au gré des situations historiques. Si d'une part, il s'efforce de définir l'Europe comme une identité culturelle porteuse de civilisation, qui suppose des acculturations nécessaires, il n'en voit pas moins d'autre part, dans les invasionsmigrations, les dangers qui en menacent la cohésion, la permanence et l'avenir. De ce point de vue, la culture européenne est en repli. Le concept des barbares opposés aux Europaioi hérité d'Hérodote reprend vie, parce que l'Europe est assiégée. Cela n'exclut pas que, pour l'humaniste, l'ancrage dans l'humanitas - dans ce lieu le plus intime à l'homme - demeure fragile, en danger de passer parfois dans ces terrae incognitae, que le Moyen Âge désignait par l'expression: "Hic sunt dracones » [ Ici sont les dragons »] (Lowe, $2013: 18$ ).

\section{BIBLIOGRAPHIE}

CHABOD Federico (2000), Europes. De l'Antiquité au XXe siècle. Anthologie critique et commentée (Y. Hersant et F. Durand-Bogaert, dir.), Paris : Laffont.

Grattarola Pio (1986), « Il concetto di Europa alla fine del mondo antico », M. Sordi (dir.), L'Europa nel mondo antico (vol. 12), Milan : Pubblicazioni della Università Cattolica del Sacro Cuore, 174-191.

LowE Keith (2013), L'Europe barbare (J. F. Hel Guedj, trad.), Paris : Perrin. (Ouvrage original publié en 2012 sous le titre Savage Continent. Europe in the Aftermath of World War II, Londres : Penguin Books.)

MESERVE Margaret (2003), « From Samarkand to Scythia: Reinventions of Asia in Renaissance Geography and Political Thought », Z. Martels (von) \& A. Vanderjagt (dir.), Pius II 'El Più Expeditivo Pontifice'. Selected Studies on Aeneas Silvius Piccolomini (1405-1464), Leiden-Boston : Brill.

Ossola Carlo (2001), Europa: miti di identità, Venise : Marsilio.

Piccolominei Aeneae Sylvii (1551), Opera omnia, Basileae : per Henricum Petri.

Piccolomini Enea Silvio (1984), Commentarii rerum memorabilium que temporibus suis contigerunt (a cura di A. van Heck, vol. II), Cité du Vatican : Biblioteca Apostolica Vaticana.

Piccolomini Enea Silvio (1991), De viris illustribus (a cura di A. van Heck), Cité du Vatican : Biblioteca Apostolica Vaticana.

Piccolomini Enea Silvio (2001), De Europa (a cura di A. van Heck), Cité du Vatican : Biblioteca Apostolica Vaticana.

Piccolomini Enea Silvio (2004), Asia (a cura di N. Casella), Bellinzona : Edizioni Casagrande. Piccolomini Enea Silvio (2005), Europa (A. Hartmann, trad.), Heidelberg-Basel : verlag regionalkultur.

Piccolomini Enea Silvio (2010), Descripcion de Asia (D. Fernandez Sanz, trad.), Madrid : CSIC. 
POLASTRON Lucien X. (2009), Livres en feu. Histoire de la destruction sans fin des bibliothèques, Paris : Gallimard.

Runciman Steven (2007), La Chute de Constantinople 1453, (H. Pignot, trad.), Paris : Tallandier. (Ouvrage original publié en 1965 sous le titre The Fall of Constantinople 1453, Cambridge : University Press).

Stolf Serge (2015), «E. S. Piccolomini et les Turcs : l'Europe face à son ennemi », Cahiers d'Études Italiennes (21), 105-117.

WOLKAN Rudolf (1918), Der Briefwechsel des Aeneas Silvius Piccolomini (vol. 68), Vienne : Fontes Rerum Austriacarum. Diplomataria et Acta.

\section{ANNEXES}

Serge Stolf est Professeur émérite d'études italiennes membre de LUHCIE, à l'Université Grenoble-Alpes. Ses travaux sont principalement orientés sur l'humanisme italien et latin des $\mathrm{XIV}^{\mathrm{e}}$ et $\mathrm{XV}^{\mathrm{e}}$ siècles (problématique des genres et de la traduction, intertextualité, ironie et dérision, rhétorique) et sur des traductions (Boccace, Piccolomini). Parmi les ouvrages récents : Les lettres et la tiare. E. S. Piccolomini, un humaniste au $\mathrm{XV}^{e}$ siècle (2012), Paris : Garnier (biographie historique) ; E. S. Piccolomini, Les Misères des gens de cour. Le songe de Fortune (2015), Grenoble : ELLUG (traduction).

\section{NOTES}

1. L'auteur cite des emplois du substantif Europenses pour désigner les Francs et leurs alliés vainqueurs des Arabes en 732 (p. 190): «diluculo prospiciunt Europenses Arabum temtoria; Europenses vero... in suas se leti recipiunt patrias (Continuatio Hispana ad ann. 754) ».

2. Le sens de cette humanitas est configuré évidemment par les paramètres des valeurs culturelles de l'humanisme (dans son sens historique), où les arts et les réalisations terrestres, cités et lois, témoignent de cette « humanisation».

3. Le seul qui soit cité, Niccolò de' Conti, explorateur vénitien, mort en 1469, affirmait avoir trouvé au pays du Cathay (Chine du Nord) des gens civilisés. Comme nous le lisons, Pie II le met en doute: "Quod si credimus magna profecto morum mutatio facta est ab his quos antiqui prodiderunt, qui Scythas ferme omnes pastorales esse affirmaverunt et propemodum intractabiles, nec horrida septemtrionis ora admittit mores, quibus vel Graecia olim claruit, vel Italia nunc floret, aut certe Cathaium ipsum quod tantopere laudant minus septentrionale est quam pictura demonstrat. "

4. « tantum [est] spacium, quod Europam Asiamque disterminat ».

5. « sed illorum origo ex Chreta atque Italia fuit ; Turcorum gens scythica et barbara est. »

6. «Doleo infinitas sanctorum basilicas opere mirando constructas vel ruine vel spurcitie Mahometi subjacere. quid de libris dicam, qui illic erant innumerabiles, nondum Latinis cogniti? heu, quot nunc magnorum nomina virorum peribunt? secunda mors ista Homero est, secundus Platoni obitus. Ubi nunc philosopharum aut poetarum ingenia requiremus? extinctus est fons musarum, utinam tantum nobis superaret ingenii, ut hanc calamitatem dignis vocibus deplorare possemus. " - Si toutes les églises ne furent pas détruites, la plupart des livres furent brûlés (Runciman, 2007 : 216-217).

7. Sur les destructions des bibliothèques dans l'histoire, voir Polastron, 2009.

8. " natio greca, quamuis olim barbara fuerit, et iterum nostro tempore deleto Grecorum imperio dominantibus Turcis in barbariam redit. » 


\section{9. «Scytharum natio barbara est. "}

10. Sur les Scythes, leur image dans l'Antiquité et l'exploitation qu'en fait Piccolomini dans l'Asia, voir Meserve, 2003 : "The prevailing spirit of the work is not one of scholarly criticism but rather of political polemic» (p. 19), jugement amplement justifié par la lecture attentive de l'Asia.

11. « Ex Asiatica Scythia migraverunt in Europam Unni, feroces populi [...] qui [...] omnem barbariem inter Thanaim ac Danubium occupavere. Ex his magnus ille Attilas ortus est qui subacta Pannonia, Italiam ingressus Aquileiam subvertit et in Germaniam transiens plurimas vastitates edidit, ac transmisso Rheno... "- Nous soulignons entre parenthèses tous les verbes latins indiquant les passages et les franchissements des territoires.

12. Voir Stolf, 2015 : 105-117. Cet article examine en particulier la dimension politique de cette «barbarisation» de l'ennemi. Nous avons volontairement laissé de côté cet aspect dans la présente contribution, considérant que cette «barbarisation » recouvre aussi une dimension de conviction personnelle sur les fondements d'une Europe comme lieu de civilisation.

13. « in Europa, id est in patria, in domo propria ». Il s'agit du discours, prononcé par Piccolomini en 1454, à la diète de Ratisbonne, sur le désastre de Constantinople et sur la nécessité de se rassembler pour faire la guerre aux Turcs (Oratio Ænece de Constantinopolitam Clade, et bello contra Turcos congregando).

14. « Extat adhuc non longe ab ortu Thanays altera Hungaria nostre huius, de qua sermone est, mater, lingua et motibus pene similis, quamuis nostra ciuilior est, Christi cultrix ; illa ritu barbarico uiuens seruit idolis. »

15. "Valachia perquam lata regio est, a Transiluanis incipiens usque euxinum protensa pelagus, plana ferme tota et aquarum indigua, cuius meridiem Hister fluuius excipit. Septentrionem Roxani occupant, quos nostra etas Ruthenos appellat, et uersus fluuium Thiram nomades, Scytharum genus, quos Tartaros hodie uocitamus. [...] sermo adhuc genti romanus est, quamuis magna ex parte mutatus et homini italico uix intelligibilis. »

16. Picccolomini, $2001: 86$ (« creberrimas mutationes »; « mira rerum mutatio »); 182 (« mutationes sub Friderico factas »).

17. " mira reum mutatio et fluxa humani imperii gloria : hec est Macedonia, que duobus inclyta regibus, Grecia Thraciaque subacta imperium protendit in Asiam [...]. hec eadem nostra etate spurcissime Turcorum genti subiecta tributum pendere et iugum ferre miserrimum cogitur. »

18. " christianarum ultima provinciarum ".

19. « christiana religio hanc orbis partem nostro generi aperuit, que ferocissimis gentibus detersa barbarie mitioris uite cultum ostendit. »

20. " ab euangelio Christi alienum est ".

21. "nostro demum seculo ad christianas manus Gebeltarum rediisse et, ubi fuere Maumethis templa, nunc ecclesias diuine pietati dicats esse et multos ex christianis habitatores eo confluxisse. »

22. "Ungari qui hactenus nostrae fidei clypeus, nostrae religionis murus fuere. »

\section{RÉSUMÉS}

Dans ses deux ouvrages, De Europa et Asia, Enea Silvio Piccolomini analyse les mouvements - invasions, migrations - des populations entre l'Orient et l'Europe. Face aux dangers que représente l'avancée turque dans l'Europe latine et grecque (prise de Constantinople), il tente de définir les ancrages qui permettront à l'Europe et à sa culture de fixer les peuples acquis à sa 
civilisation. Les frontières ainsi définies - moins politiques et géographiques que culturelles ne sont pas figées, mais mouvantes, faites de replis et d'expansion. Définir une identité devient nécessaire dans une histoire où les ancrages se révèlent parfois fragiles.

In his two works, De Europa and Asia, Enea Silvio Piccolomini analyzes the movements-invasions, migrations-of populations between the East and Europe. In the face of the dangers which the Turkish advance in Latin and Greek Europe represents (after the fall of Constantinople), he tries to define points of anchorage which will allow Europe and its culture to fix the people who have chosen his civilisation. The borders thus defined -less political or geographical than culturalare not fixed, but moving, made of withdrawal and of expansion. To define an identity becomes necessary in a historical period during which roots appear sometimes fragile.

\section{INDEX}

Keywords : Europe, migrations, invasions, anchoring, roots, borders, frontiers, identity, civilisation

Mots-clés : Europe, migrations, invasions, ancrages, frontières, identité, civilisation

\section{AUTEUR}

\section{SERGE STOLF}

Professeur émérite à l'Université Grenoble-Alpes (LUHCIE). 